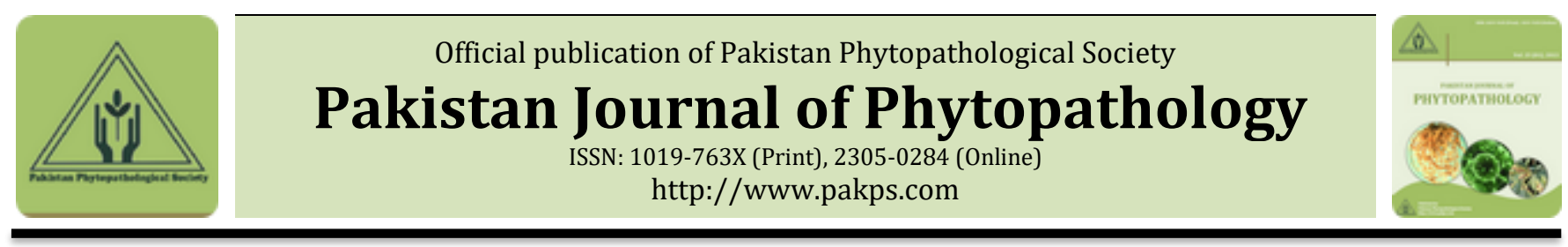

\title{
MORPHOLOGICAL RESPONSES OF KING OYSTER MUSHROOM AGAINST AUGMENTATION OF COTTON WASTE WITH CITRUS PEEL
}

\author{
aMuhammad M. Jahangir*, aMuhammad A. Khatana, bNasir A. Khan, cGhufrana Samin, aKhurram Ziaf, \\ aMuhammad U. Farooq, aWaseem Iqbal \\ a Institute of Horticultural Sciences, University of Agriculture, Faisalabad, Pakistan. \\ $\boldsymbol{b}$ Department of Plant Pathology, University of Agriculture, Faisalabad, Pakistan \\ c Department of Chemistry, University of Engineering and Technology Lahore, Faisalabad Campus, Pakistan.
}

\begin{abstract}
A B S T R A C T
The utilization of agro-industrial waste materials for production of mushrooms has become a crucial research precedence recently. Pleurotus eryngii is considered one of the most famous mushrooms because of nutraceutical and high nutritional value. Therefore, a study was conducted in Medicinal and Mushroom Lab to evaluate the morphological attributes of king oyster mushroom against cotton waste (CW) and citrus peel (CP) based substrates. $\mathrm{CW}$ alone and its different combinations with CP i.e. T0: CW (100 \%), T1: CW (98\%) + CP (2\%), T2: CW (96\%) + CP (4\%), T3: CW (94\%) + CP (6\%), T4: CW (92\%) + CP (8\%) and T5: CW (90\%) + CP (10\%) were used in experiment. The experiment was conducted by applying 2-factor factorial under completely randomized design with 5 replications. Different morphological attributes viz. spawn running and mycelium growth initiation, duration to complete mycelium growth (days), pinhead development, pinheads/bag, duration to complete flushes $\left(1^{\text {st }}, 2^{\text {nd }}, 3^{\text {rd }}\right)$, mushroom fresh weight, yield/bag and biological efficiency were calculated. Results were analyzed by ANOVA and mean values are equated by LSD test at $5 \%$ probability level. Results indicated that spawn running, the growth of mycelium, duration to complete flushes, pinhead development and duration, yield and biological efficiency all were significantly influenced by treatment T2 (CW 96\% + CP 4\%) as compared to control. Overall results revealed that combinations of citrus peel and cotton waste can be utilized as a promising substrate for king oyster mushroom production.
\end{abstract}

Keywords: mushroom morphology, substrates, mycelium growth, flushes, biological efficiency.

\section{INTRODUCTION}

King oyster mushroom belongs to family Agaricaceae and native of Mediterranean regions of Africa and Europe. It is commercially grown in various regions of the world including many countries of Asia due to its unique texture, flavor and nutritious value. It is considered as an important mushroom among Pleurotus spp because of good shelf life, stipe, pileus and culinary qualities. As for as morphology is concerned, Pileus color ranges from gray to brownish gray, 2 to $8 \mathrm{~cm}$ wide, stipe 0.8 to $4 \mathrm{~cm}$ thick and 3.8 to $14 \mathrm{~cm}$ long (Yildiz et al., 2002; Shah et al., 2004).

Submitted: May 13, 2018

Revised: June 06, 2018

Accepted for Publication: June 13, 2018

* Corresponding Author:

Email: muzammil_jahangir@hotmail.com

(C) 2017 Pak. J. Phytopathol. All rights reserved.
Many environmental and physical factors are involved in king oyster mushroom production which includes spawn, growing media, light, temperature, moisture and $\mathrm{pH}$. Temperature has a great impact on enzymatic action and vitamins incorporation that may enhance or suppress developmental process (Bing et al., 2010). King oyster mushroom required $90-95 \%$ humidity, 2000 relative $\mathrm{CO}_{2}$, $16-22^{\circ} \mathrm{C}$ temperature and 500-1000 lux light for growth of fruiting body. Mycelium growth is completed in 55 to 60 days and 3 to 4 flushes produced in this mushroom (Chen et al., 2004).

Numerous agricultural waste materials are being used for the cultivation of Pleurotus eryngii which includes rice straw, wheat straw, sugarcane bagasse, cotton ginning waste, soybean stalks, cotton seed hulls, wheat bran, corn cobs, lime and maize powder etc. production cycle includes spawn preparation, spawn inoculation, spawn 
running, mycelium growth, pinheads and fruiting body development, stipe and pileus development. Spawn quality and type (solid or liquid) influence the mycelium growth and production of mushroom (Mamiro and Royse, 2008).

After citrus juice extraction during processing many byproducts are produced which includes pulp, seed and peel which are utilized as animal feed or for pectin extraction. These by-products can be utilized as substrates for crop production. Numerous protein, antioxidants, prebiotics, ethanol, enzymes, organic acids and polysaccharides are obtained from these by-products (Mamma and Christakopoulos, 2008). Nutrients examination of citrus peel showed that it contained sugars $(16.8 \%)$, cellulose (9.4\%), lignin (0.9\%), hemicellulose $(10.8 \%)$, pectin (42.8\%), starch (3.8\%), ashes (3.8\%), proteins (6.8\%) and fats (1.10\%) (Rivas et al., 2008).

Cotton waste is being used commercially for the production of king oyster mushroom production in Pakistan, but year-round cotton waste is not available. Citrus is major fruit crop of Pakistan and a plenty of citrus waste is produced in our country. There is a little knowledge about utilization of citrus peel for Pleurotus eryngii cultivation that's why a study was designed to check the efficacy of citrus peel and cotton waste for the cultivation of Pleurotus eryngii mushroom.

\section{MATERIALS AND METHODS}

The present study was performed at Medicinal \& Mushroom Lab, Institute of Horticultural Sciences, University of Agriculture, Faisalabad during 2016-2017 for production of Pleurotus eryngii strains "P10 (China) and P11 (Canada)" by utilizing cotton waste alone and its different combinations with citrus peel as a basal media.

Preparation and bag filling of Substrate: For substrates preparation, citrus peel and cotton waste were soaked in water and spread on floor separately. Than $4 \%$ lime was added and a heap was made of both materials separately and covered the heaps with a polythene sheet. After two days both substrates were spread on the ground for evaporation of excess water. Then after fermentation process make different formulations of cotton waste and citrus peel and bags ( $6 " \times 10 ")$ were filled and mouths of bags were tied by rubber bands (Khan $e t$ al., 2017).

Pasteurization and spawning of bags: For pasteurization, Bags were placed in a drum and steam was produced by heating the water present in the drum at a temperature of $50-65{ }^{\circ} \mathrm{C}$ for 2 to 3 hours. After that bags were placed overnight under shade to cool down. The weight of each bag was $500 \mathrm{~g}$ and spawn was added @ $1 \%$ of weight of the substrate.

Control of temperature and Humidity: Growth room temperature was maintained between 18 to $22{ }^{\circ} \mathrm{C}$ during spawn running while humidity was sustained at $70-80 \%$ by applying water on the ground. For cropping, temperature was maintained at $16-19{ }^{\circ} \mathrm{C}$. Fructification was started after completion of mycelium growth then humidity of room was maintained between 80 to $90 \%$ by sprinkling water on floor and bags (Sardar et al., 2017).

Parameters: Different morphological parameters viz. duration required to initiate spawn running (days), duration to complete mycelium growth (days), duration for initiation of pinheads (days), pinheads/bag, flushes/bag, duration to complete flushes (first, second and third), yield/bag and biological efficiency (\%) were recorded.

B.E $(\%)=$ (fresh weight of mushroom per bag / dry weight of substrate per bag) $\times 100$

Statistical procedure: The experiment was conducted by adopting 2- factorial under CRD with 5 replicates. Results were scrutinized by ANOVA techniques and mean values were equated by using LSD test at $5 \%$ level of significance (Steel et al., 1997).

\section{RESULTS}

Results regrading morphological parameters showed that citrus peel had non-significant effect on spawn running initiation time. Spawn running of each treatment was started almost at the same time (Fig. 1). Mycelium growth is significantly affected by cotton waste and citrus peel based substrates. Maximum duration (23.10 days) for the fulfillment of $25 \%$ growth was observed at T5: CW $(90 \%)+\mathrm{CP}(10 \%)$ and minimum (16.40 days) was at T2: CW (96\%) + CP (4\%) (Fig. 2). For the accomplishment of $50 \%$ mycelium growth maximum (35.30 days) duration was observed at T0: CW (100 \%) and minimum (25.35 days) was at T2: CW (96\%) + CP (4\%) (Fig. 3). Maximum (49.75 days) duration for $75 \%$ mycelium growth fulfillment was obtained at T4: CW (92\%) + CP (8\%) and minimum (39.25 days) was at T2: CW (96\%) + CP (4\%) (Fig. 4). For the $100 \%$ fulfillment of mycelium growth maximum (56.35 days) time period was observed at T4: CW $(92 \%)+\mathrm{CP}(8 \%)$ and minimum (49.30 days) was at T2: CW (96\%) + CP (4\%) (Fig. 5). Pinhead formation duration was maximum (69.35 days) at T4: CW (92\%) + CP (8\%) and minimum (59.15 days) at T2: CW (96\%) + CP (4\%) (Fig. 6). 


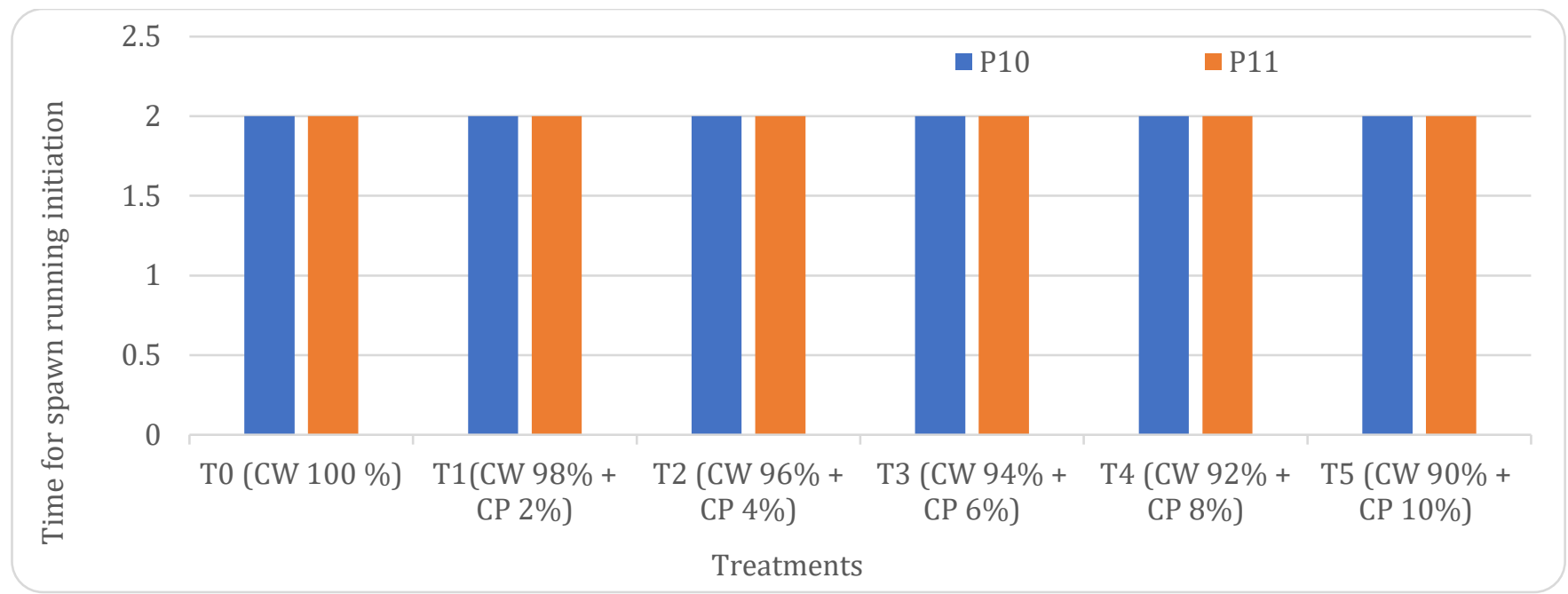

Figure 1. Spawn running initiation in response to cotton waste and citrus peel.

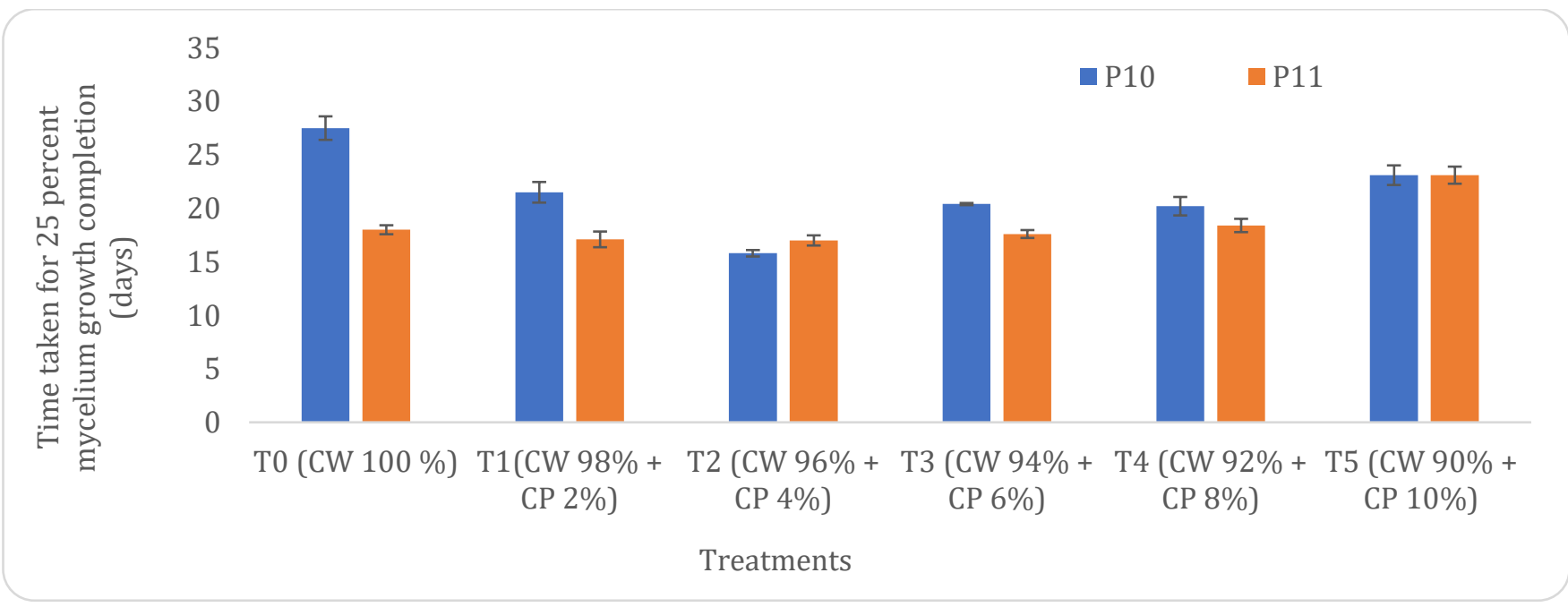

Figure 2. $25 \%$ Mycelium growth completion in response to cotton waste and citrus peel based substrates.

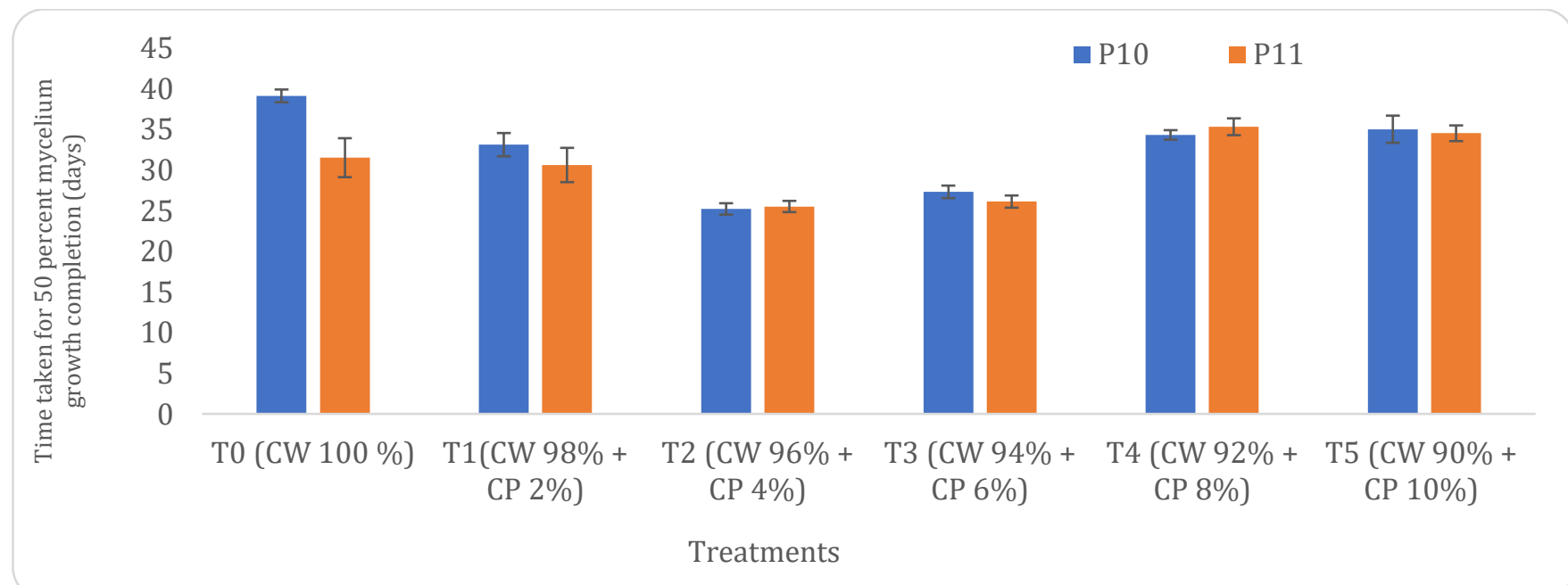

Figure $3.50 \%$ Mycelium growth completion in response to cotton waste and citrus peel based substrates. 


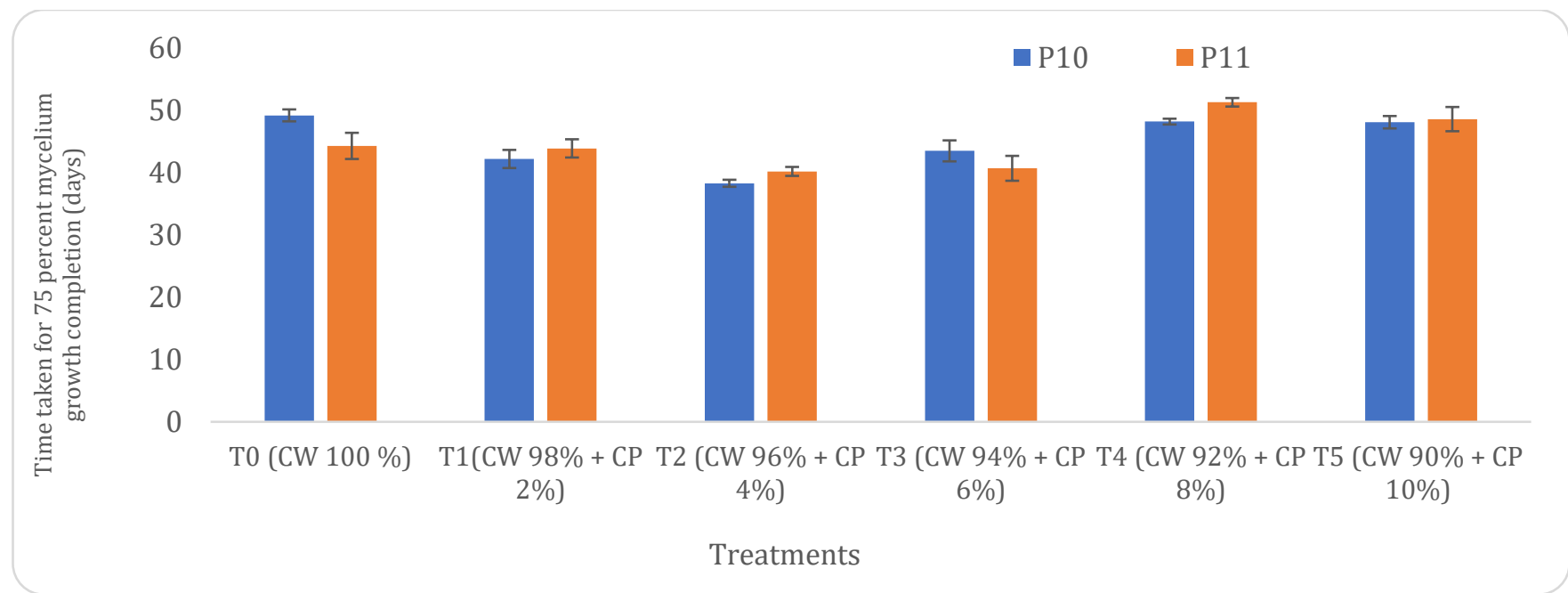

Figure 4. 75 \% Mycelium growth completion in response to cotton waste and citrus peel based substrates.

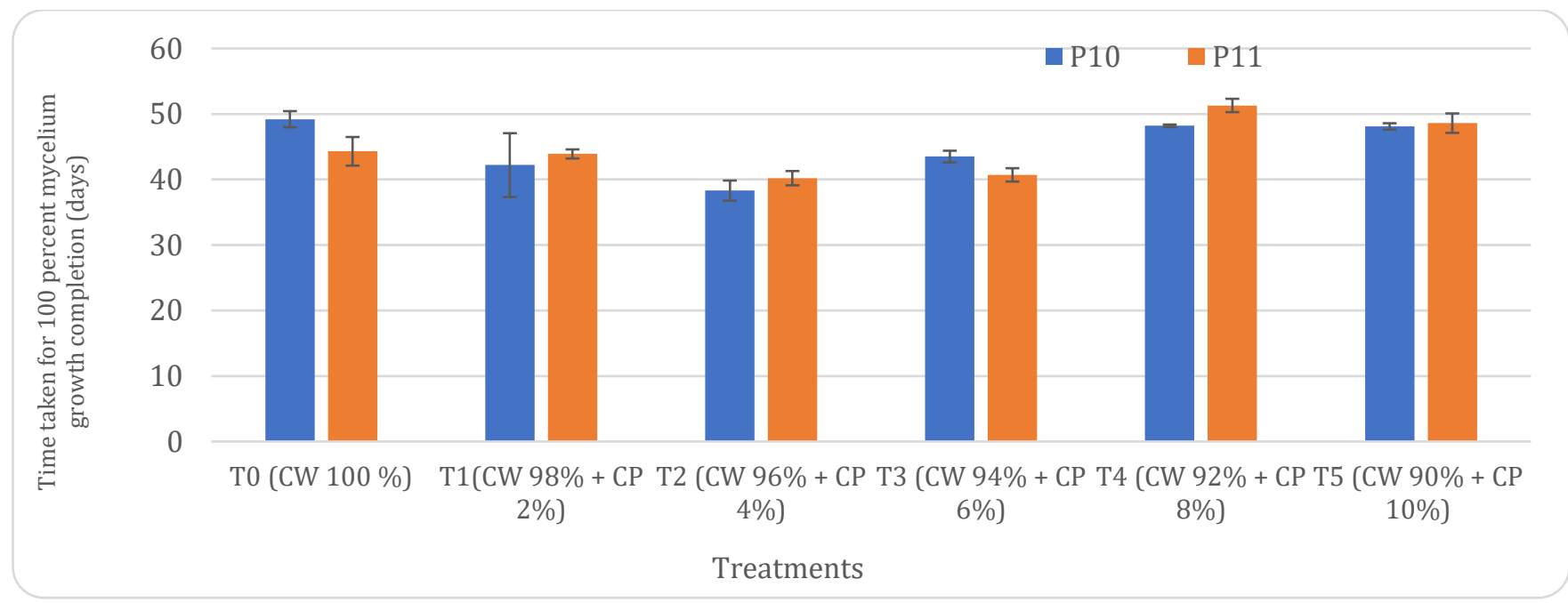

Figure $5.100 \%$ Mycelium growth completion in response to cotton waste and citrus peel based substrates.

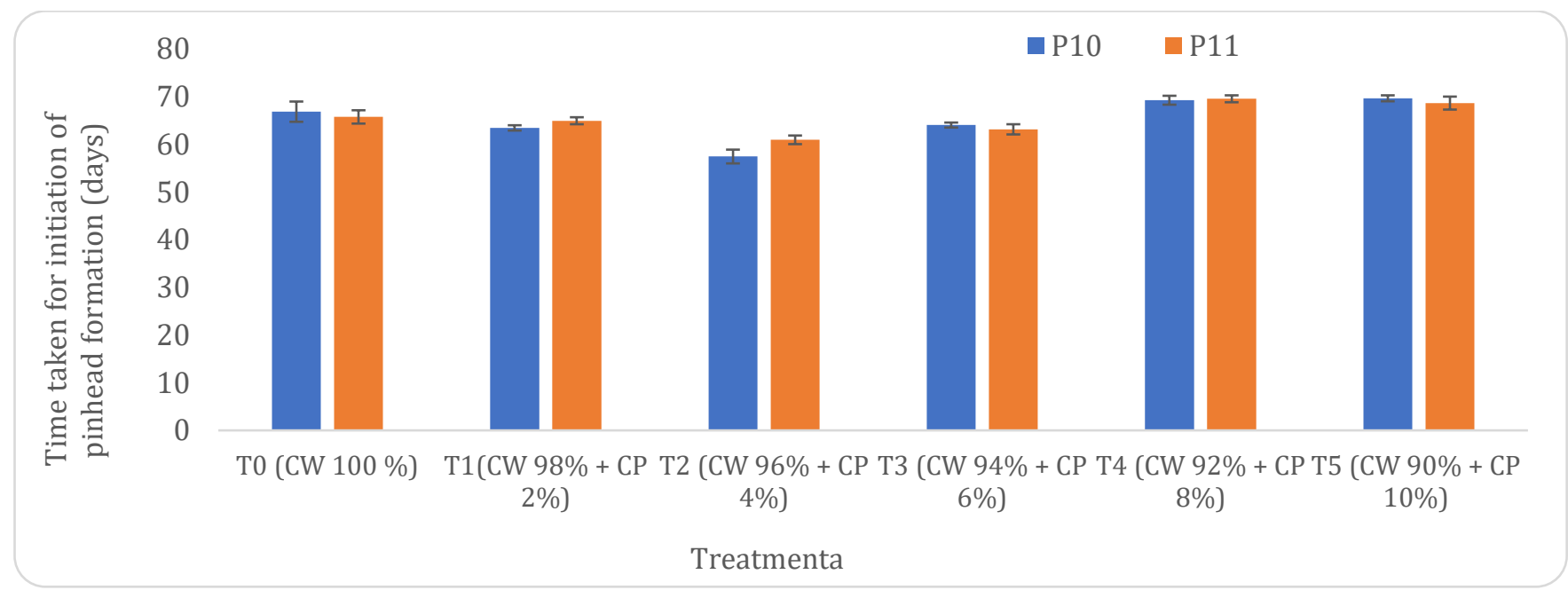

Figure 6. Initiation of pinheads in response to cotton waste and citrus peel based substrates. 
Maximum (32.10) number of pinheads per bag were observed at T2: CW (96\%) + CP (4\%) and minimum (7.3) at T5: CW (90\%) + CP (10\%) (Fig. 7).

Maximum (6) number of flushes were obtained at T2: CW $(96 \%)+\mathrm{CP}(4 \%)$ and minimum (3.5) was at T0: CW (100 \%) (Fig. 8).

Time required for completion of $1^{\text {st }}$ flush was observed maximum (79.41 days) at T4: CW (92\%) + CP (8\%) and minimum (69.06 days) at T2: CW (96\%) + CP (4\%) (Fig. 9), for $2^{\text {nd }}$ flush was maximum (91.41 days) at T4: CW $(92 \%)+\mathrm{CP}(8 \%)$ and minimum (81.06 days) at T2: CW (96\%) + CP (4\%) (Fig. 10) and for $3^{\text {rd }}$ flush maximum (105.42 days) was at T4: CW (92\%) + CP (8\%) and minimum (95.07 days) was at T2: CW (96\%) + CP (4\%)
(Fig. 11).

Fresh weight of mushroom for $1^{\text {st }}$ flush was obtained maximum (123.55g) at T2: CW (96\%) + CP (4\%) and minimum (33.82 g) was at T4: CW (92\%) + CP (8\%) (Fig. $12)$, for $2^{\text {nd }}$ flush it was maximum $(95.91 \mathrm{~g})$ at $\mathrm{T} 2$ : $\mathrm{CW}$ $(96 \%)+\mathrm{CP}(4 \%)$ and minimum $(30.73 \mathrm{~g})$ at T0: CW (100 \%) (Fig. 13) and for $3^{\text {rd }}$ flush weight was maximum (68.39 g) at T2: CW (96\%) + CP (4\%) and minimum (26.42 g) was at T0: CW (100\%) (Fig. 14).

Maximum (287.85 g) yield was observed at T2: CW (96\%) + CP (4\%) and minimum (91.95 g) was at T0: CW (100\%) (Fig. 15). Biological efficiency percentage was maximum $(82.24 \%)$ at $\mathrm{T} 2$ : CW (96\%) + CP (4\%) and minimum $(26.27 \%)$ at T0: CW (100 \%) (Fig. 16).

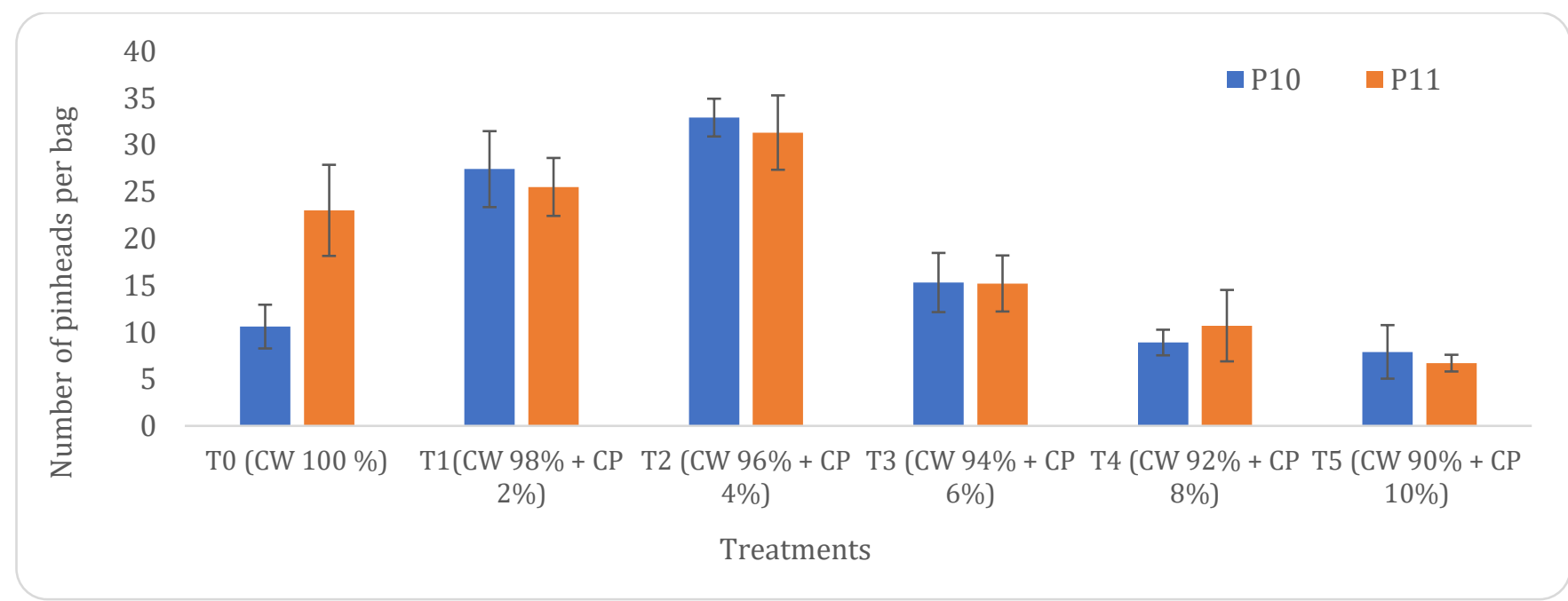

Figure 7. Number of pinheads in response to cotton waste and citrus peel based substrates.

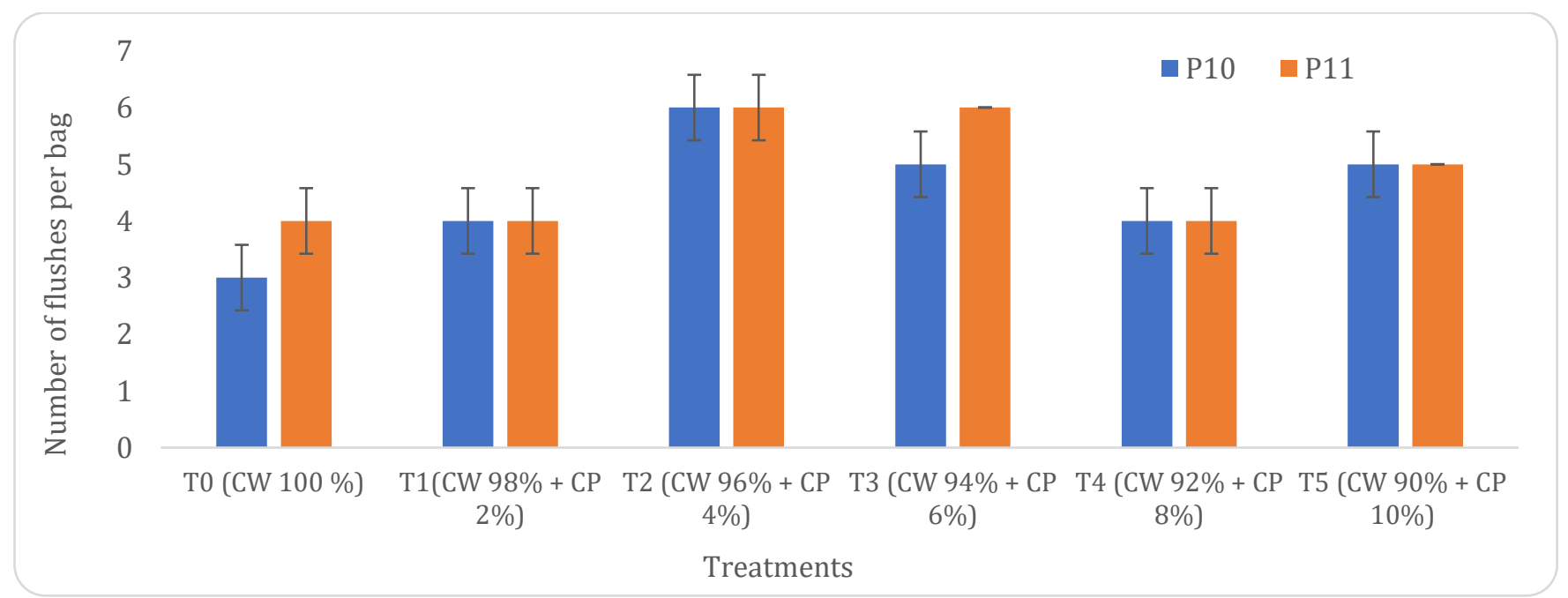

Figure 8. Number of flushes in response to cotton waste and citrus peel based substrates. 


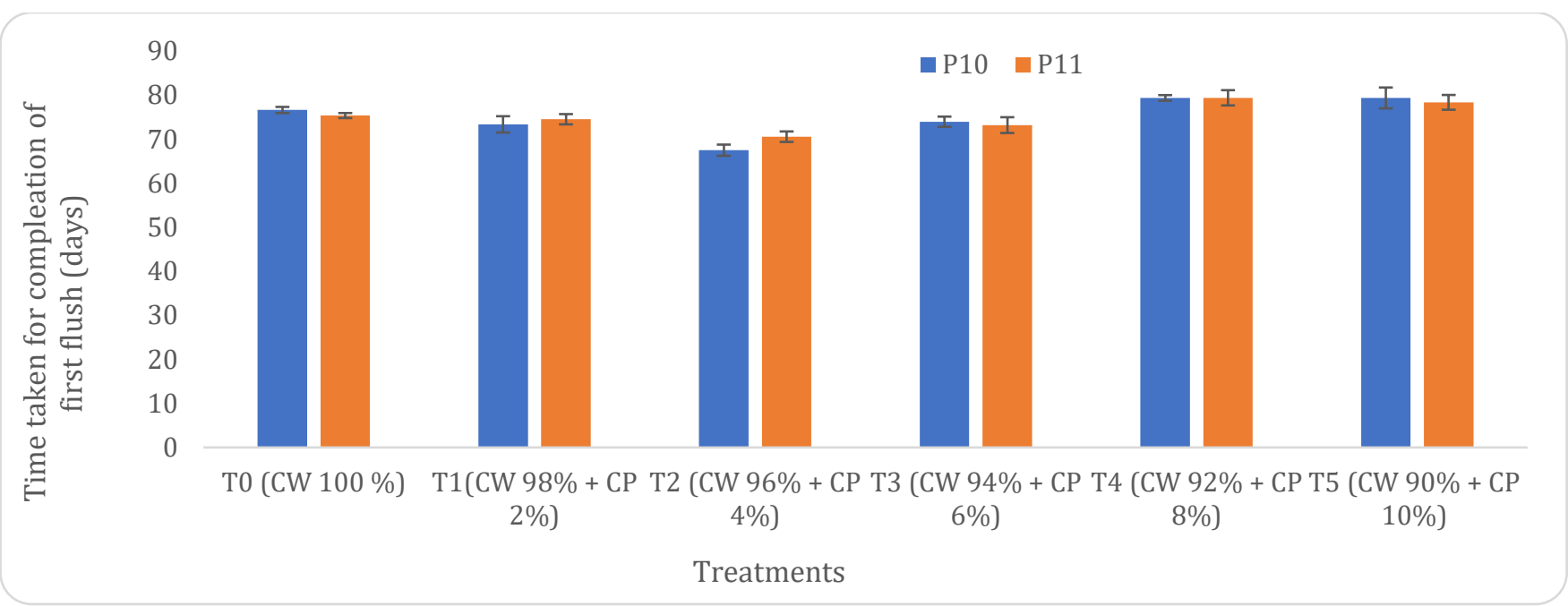

Figure 9. Completion duration of $1^{\text {st }}$ flush in response to cotton waste and citrus peel based substrates.

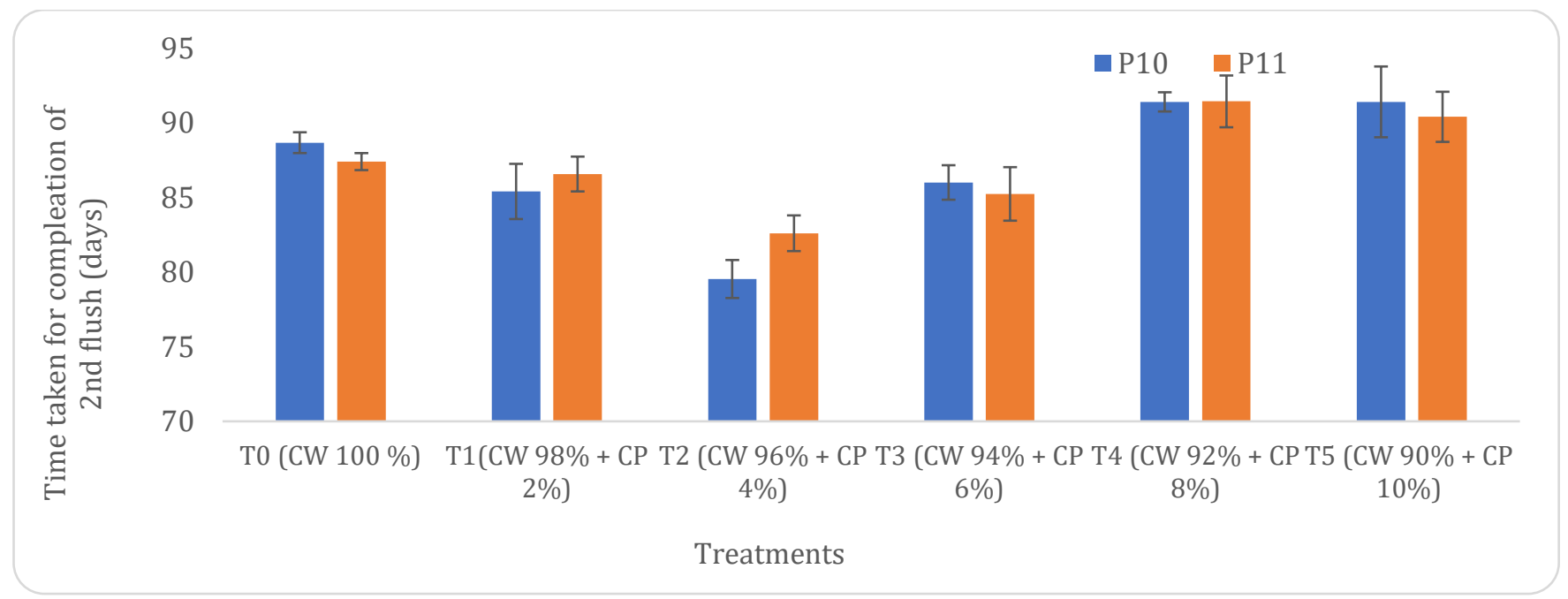

Figure 10. Completion duration of $2^{\text {nd }}$ flush in response to cotton waste and citrus peel based substrates.

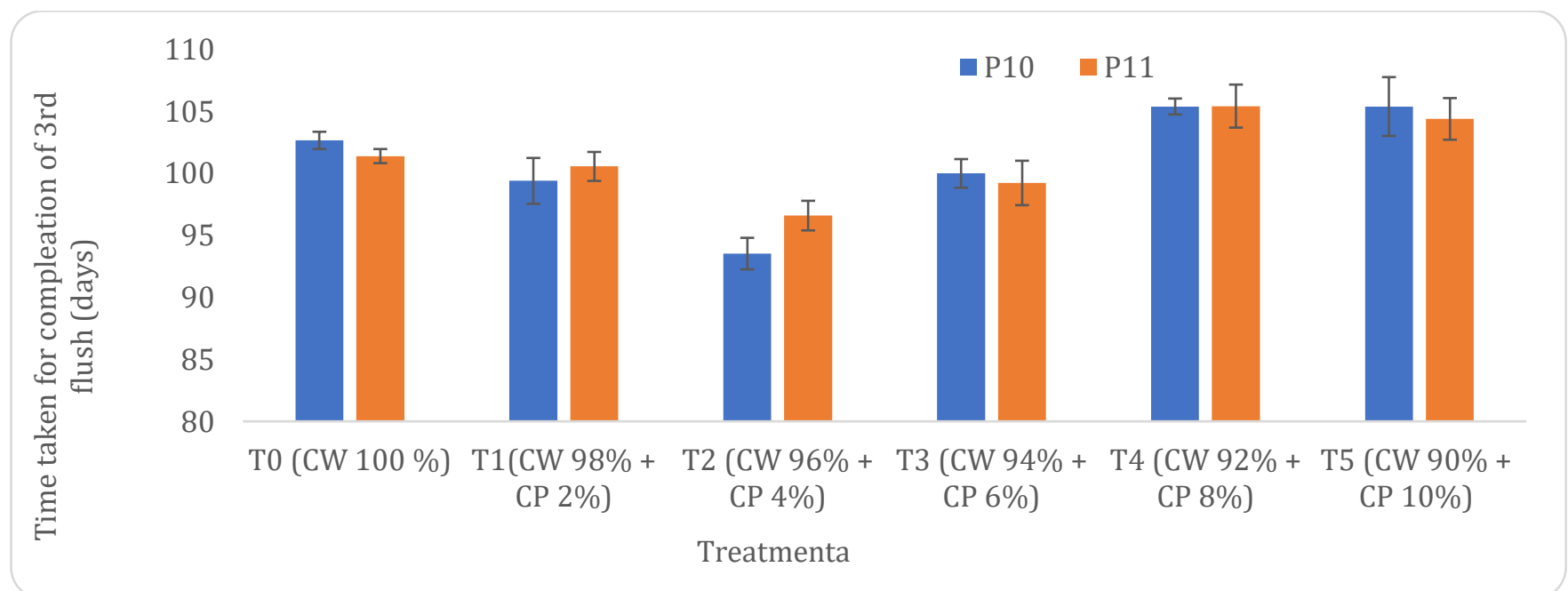

Figure 11. Completion duration of $3^{\text {rd }}$ flush in response to cotton waste and citrus peel based substrates. 


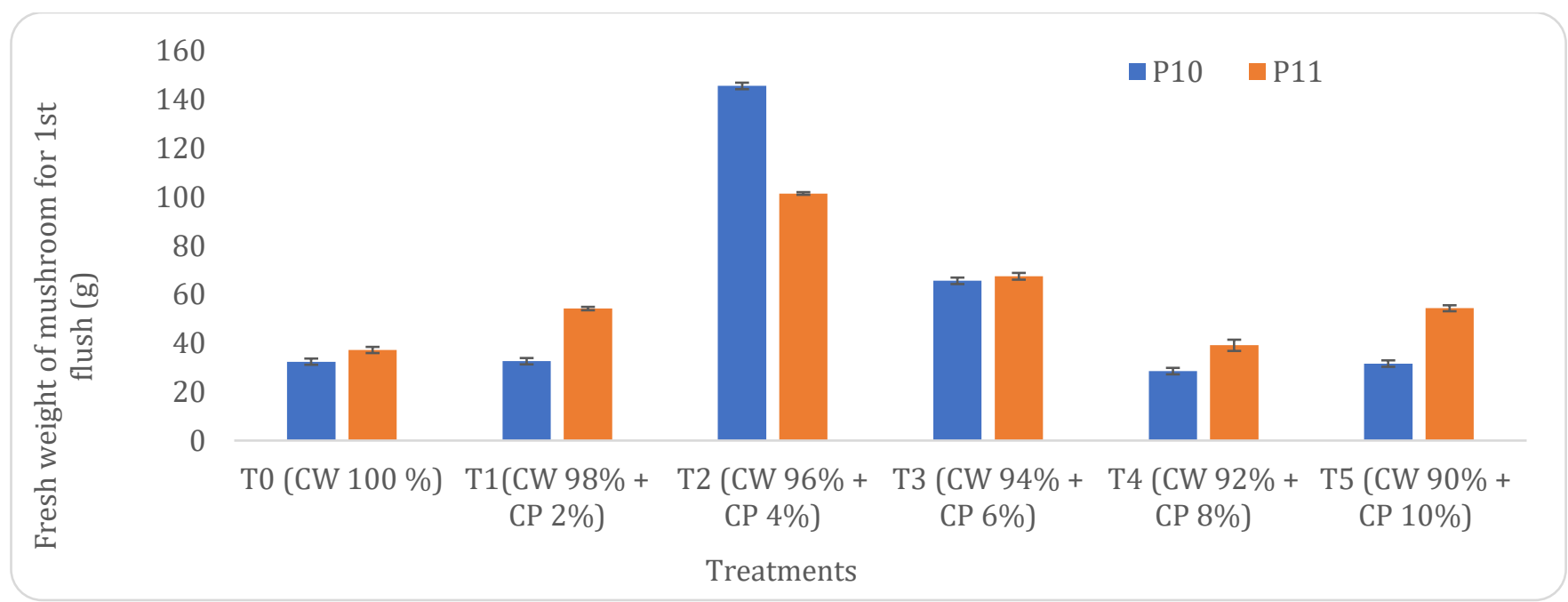

Figure 12. Weight of $1^{\text {st }}$ flush in response to cotton waste and citrus peel based substrates.

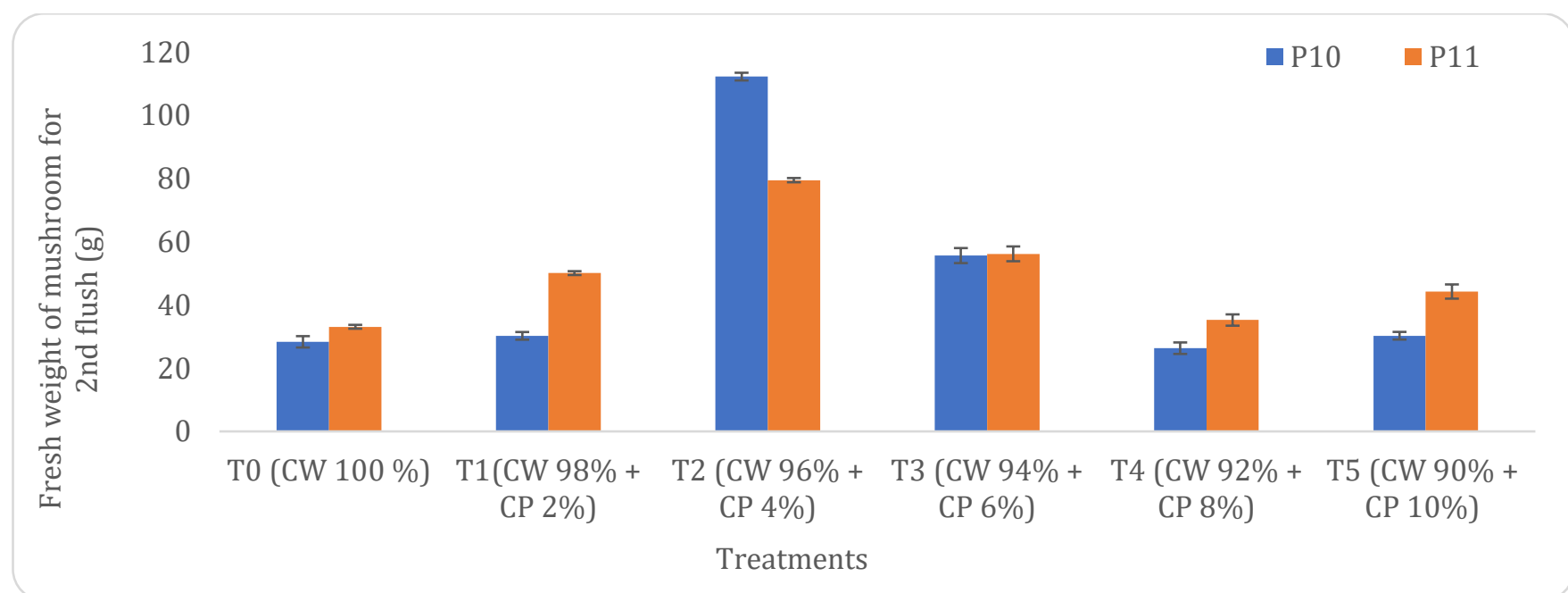

Figure 13. Weight of $2^{\text {nd }}$ flush in response to cotton waste and citrus peel based substrates.

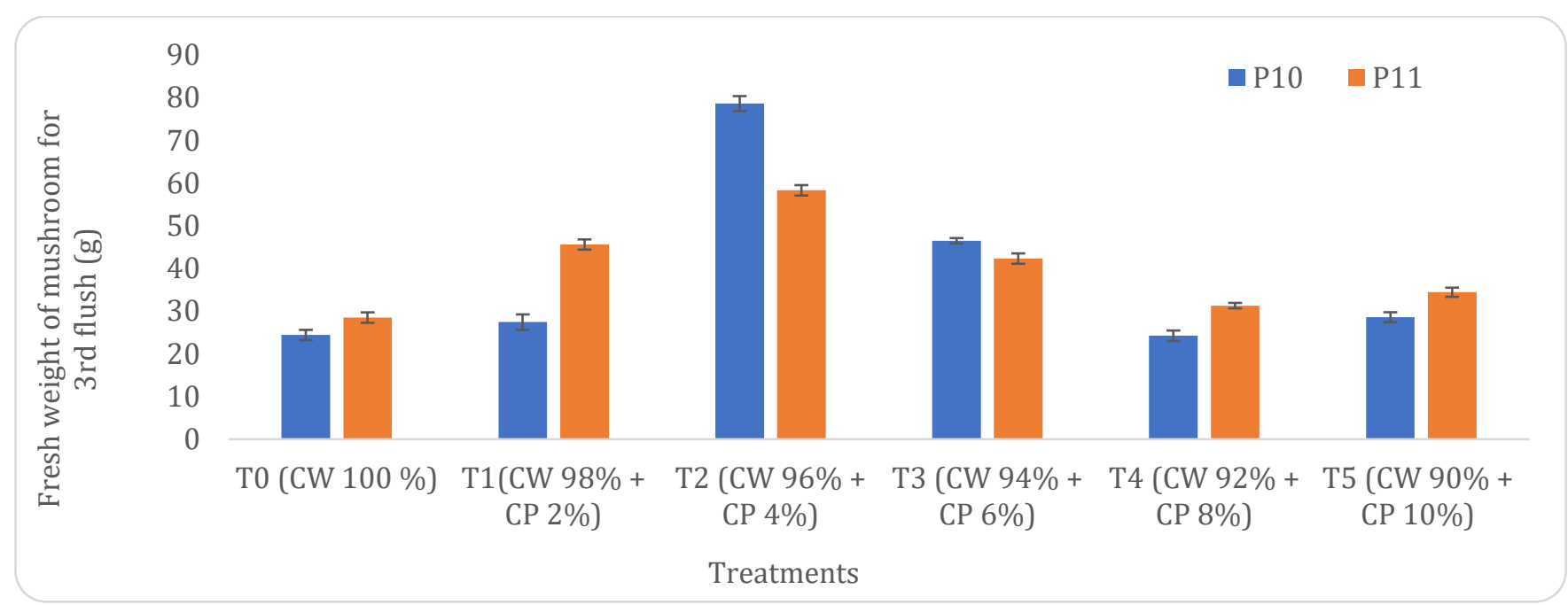

Figure 14. Weight of $3^{\text {rd }}$ flush in response to cotton waste and citrus peel based substrates. 


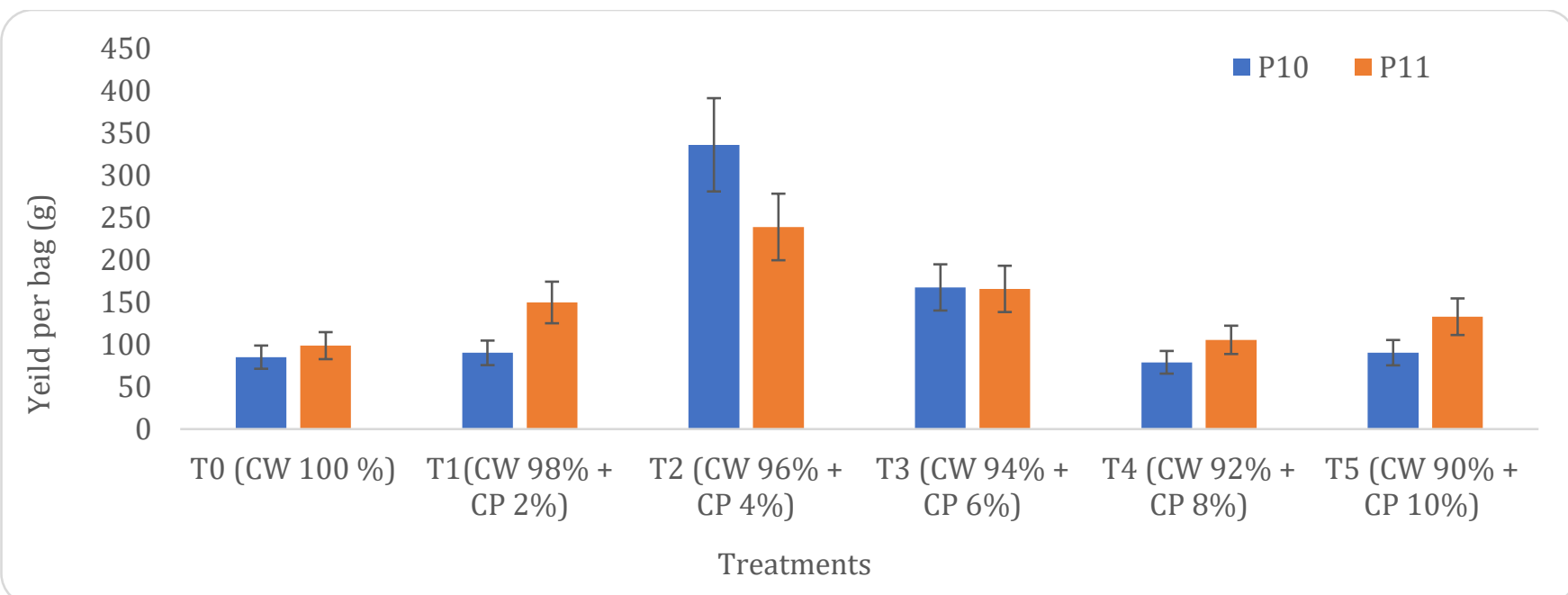

Figure 15. Yield in response to cotton waste and citrus peel based substrates.

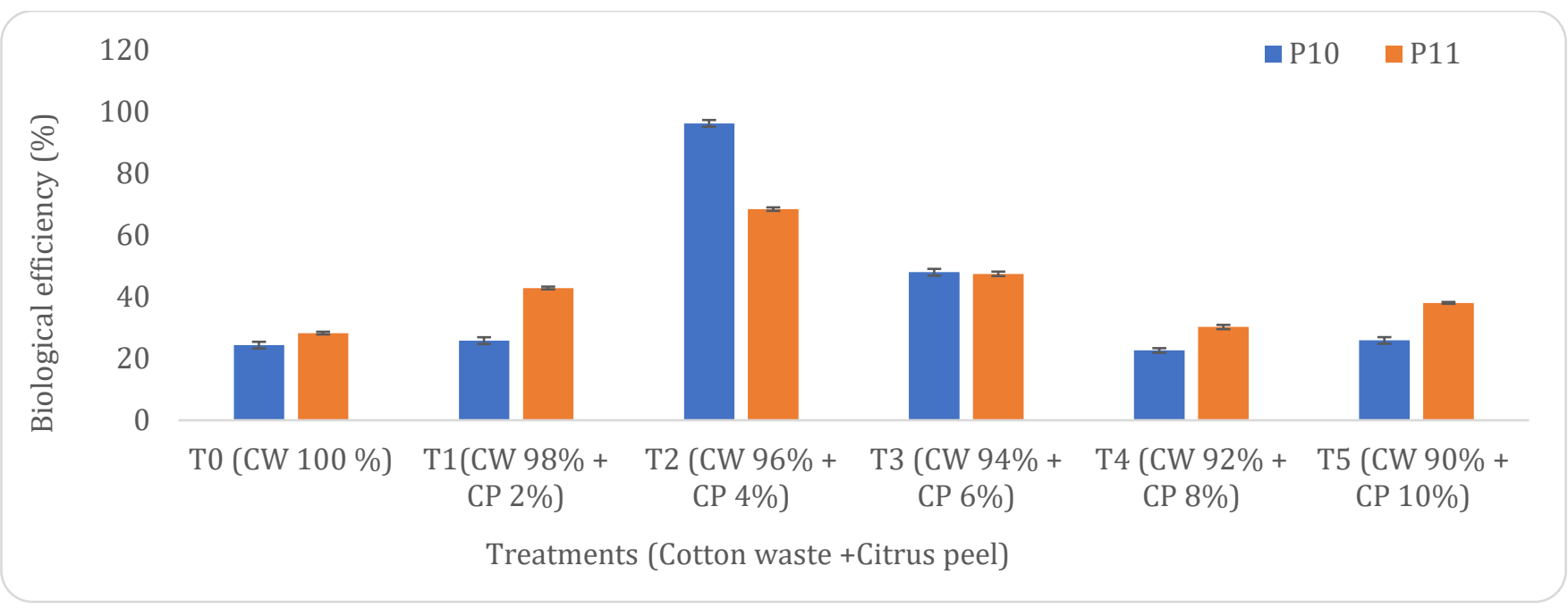

Figure 16. Biological efficiency in response to cotton waste and citrus peel based substrates.

\section{DISCUSSION}

The current research was performed to evaluate the efficacy of cotton waste augmentation with citrus peel for the cultivation of Pleurotus eryngii. Findings of our experiments are in accordance with the outcomes of Hassan et al., 2010 who reported that spawn running of different strains of king oyster mushroom are influenced by temperature, light, relative humidity, growing substrate, $\mathrm{CO}_{2}$, nutrients and $\mathrm{O}_{2}$. Akyuz and Yildiz, 2008 stated that moisture is an important factor for the growth of Pleurotus eryngii production because it directly affected the production of mushroom. Pinheads formation influenced by environmental conditions of growth room i.e. temperature, light, humidity, substrate chemical and physical composition and spawn. The optimal temperature for pinhead formation is $16-22^{\circ} \mathrm{C}$. Direct application of water after pinhead formation should be avoided because it caused damage to pinheads (Singh et al., 2011). Number of flushes depends upon numerous environmental factors i.e. temperature, humidity and light and on cultural practices, growing media and spawn quality. These factors enhanced and suppressed the number of flushes depending upon their optimal conditions. Dead pinheads on substrates also have a bad impact on mushroom yield so removal of these pinhead is necessary for better yield (Stamets and Chilton, 1983). Physical and chemical characteristics of growing media effect the yield of mushroom. By the addition of different chemicals and supplements in substrates (Esterda and Royse, 2007). Biological 
efficiency is the mushroom's weight in accordance with weight (dry) of growing substrate. Biological efficiency of king oyster mushroom enhanced by the addition of different nutrients and chemicals (Kirbag and Akyuz, 2008).

\section{REFERENCES}

Ahmed, S.A., J.A. Kadam, V.P. Mane, S.S. Patil and M.M.V. Baig. 2009. Biological efficiency and nutritional contents of Pleurotus florida (Mont.) Singer cultivated on different Agro-wastes. Nat. Sci. 7(1): 44-48.

Akyuz, M and A. Yildiz. 2008. Evaluation of cellulosic wastes for the cultivation of Pleurotus eryngii (DC. ex Fr.) Quel. African J. Biotech. 7(10): 1494-1499.

Bing, C.X., W.D. Zhong, Z.M. Bin and G.X. Ping. 2010. Effects of different external environment on the mycelium growth of Pleurotus eryngii. J. Anhui Agric. Uni. 1: 123-128.

Chen, A.W. 2004. Oyster mushroom cultivation (Handbook by mushroom growers) Chap. 3, ISSN 1739-1377.

Hassan, F.R.H., M.G. Medany and S.D.A. Hussein. 2010. Cultivation of the King Oyster Mushroom (Pleurotus eryngii) in Egypt. Aust. J. Basic Appl. Sci. 4(1): 99-105.

Khan, N.A., W. Ahmed, A. Rehman, M.M. Jahangir and B. Khan. 2017. Efficiency of oyster mushroom (Pleurotus columbinus-P 8) using date palm leaves with combination of wheat straw and cotton waste for its yield improvement. Pak. J. Bot. 49(6): 24592464.

Kirbag, S and M. Akyuz. 2008. Effect of various agroresidue on growing periods, yield and biological efficiency of Pleurotus eryngii. J. Food Agric. Environ. 6: 402-405.

Mamiro, D.P. and D.J. Royse. 2008. The influence of spawn type and strain on yield, size and mushroom solids content of Agaricus bisporus produced on noncomposted and spent mushroom compost.
Bioresour. Technol. 99: 3205-3212.

Mamma, D. and P. Christakopoulos. 2008. Citrus peels: An excellent raw material for the bioconversion into value-added products. J. Sci. Biotechnol. 166: 358364.

Manzi, P., S. Marconi. A. Aguzzi, and L. Pizzoferrato. 2004. Commercial mushrooms: nutritional quality and effect of cooking. Food Chem. 84: 201-206.

Rivas, B., A. Torrado, P. Torre, P. Converti and A. Dominguez. 2008. Submerged citric acid fermentation on orange peel autohydrolysate. Agric. J. Food Chem. 56: 2380-2387.

Sardar, H., M.A. Ali, M.A. Anjum, F. Nawaz, S. Hussain, S. Naz and S.M. Karimi. 2017. Agro-industrial residues influence mineral elements accumulation and nutritional composition of king oyster mushroom (Pleurotus eryngii). Sci. Hort. 225: 327334.

Shah, Z.A., M. Ashraf and C.M. Ishtiaq. 2004. Comparative study on cultivation and yield performance of oyster mushroom (Pleurotus ostreatus) on different substrates (wheat straw, leaves, saw dust). Pak. J. Nutr. 3: 158-160.

Singh, M., B. Vijay, S. Kamal and G.C. Wakchaure. 2011. Mushroom cultivation, marketing and consumption. Directorate of mushroom research (ICAR).

Stamets, P and J.S. Chilton. 1983. The mushroom cultivator a practical guide to growing mushrooms at home. p 149-150. Agarikon Press Olympia., Washington.

Steel, R.G.D., J.H. Torrie and D.A. Deekey. 1997. Principles and procedures of statistics. A biometrical approach (3rd ed). McGraw Hill Book Co., Inc., NY, USA.

Yildiz, S., U.C. Yildiz, E.D. Geze and A. Temiz. 2002. Some lignocellulosic wastes used as raw material in cultivation of the Pleurotus ostreatus culture mushroom. Proc. J. Bio. Chem. 38: 301-306. 